\title{
Interventional Treatment of latrogenic False Aneurysm of Subclavian Artery Combine with Arteriovenous Malformation
}

\section{Xiaolong Zhao', Chengjian Sun ${ }^{1}$, Wei Zhang ${ }^{1}$, Hongyu Wang ${ }^{1}$, Shui Yư ${ }^{1}$, Liming Shao ${ }^{1}$}

${ }^{1}$ QingDao University ningxia road number 308 shinan district, China

\begin{abstract}
Subclavian artery pseudo-aneurysm is a rare disease, we report a case combine with arteriovenous malformation. She received failed covered stent implantation in subclavian artery before interventional embolization. And we makes a brief literature review on interventional therapy of false aneurysm of subclavian artery.
\end{abstract}

Keywords: Pseudo-aneurysm, AVM, arteriae subclavia, embolotherapy, Onyx

\section{Introduction}

Subclavian artery pseudo aneurysm is a rare disease, due to its special location, usual result is to compress the neck vessels and nevus, which will leads to a series of serious complications. The vascular injury caused by iatrogenic operation, such as subclavian vein puncture, acupuncture and so on, is occasional reported. ${ }^{2,13}$ The treatment of Subclavian artery pseudo aneurysm includes surgical treatment and endovascular treatment. Different endovascular treatments can be selected according to the location of the lesion, such as covered stents implantation and false aneurysm embolization, in order to repair of damaged blood vessels, to achieve the purpose of curing the false aneurysm. ${ }^{11}$

\section{Case report}

A 28 years old female patient had a cervical mass 10 hours. She received acupuncture, cupping therapy at a clinic 10 hours ago, due to her neck discomfort. After therapy, she felt neck swelling pain, dyspnea, and right side of her neck was swelling. The local doctors considered her in a critical condition after preliminary examination, and recommended her family members to referral to the superior hospital.

The patient came to our hospital for emergency treatment. CT scan revealed a huge soft tissue profile on the right side neck of the patient, considered as a hematoma (fig. 1). The patient was suddenly unconscious, and her heart rate and blood oxygen saturation decreased. We gave her a respirator assisted breathing therapy and MDT.

The second day after patient was hospitalized, she received vascular angiography in vascular surgery of our hospital, and diagnosed right subclavian artery arteriorrhexis proximal vertebral artery origin (fig. 2). So they performed the false aneurysm embolization, through super selective catheterization to inserted catheter into false aneurysm, and embolized with GDC. But the GDC float away. Then they tried percutaneous

This article is published under the terms of the Creative Commons Attribution License 4.0 Author(s) retain the copyright of this article. Publication rights with Alkhaer Publications. Published at: http://www.ijsciences.com/pub/issue/2017-03/ DOI: 10.18483/ijSci.1200; Online ISSN: 2305-3925; Print ISSN: 2410-4477 
subclavian artery stenting to implanted an $6 \mathrm{~mm} \times 100 \mathrm{~mm}$ covered stent(VIABAHN), and dilated stent by balloon catheter (fig. 3). After stenting, the patient was transferred to the surgical operation room to receive exploratory operation. During operation, they cut the laminasuperficialis along the anterior margin of the left sternocleidomastoid muscle, and found out lots of dark red blood clots as well as many prevertebral active bleeding points diagnosed vascular malformation and arteriovenous fistula. Due to large amount of bleeding in operation, difficult to stop bleeding, they pressure dressed wound and transferred to ICU to continue medical treatment.

The patient was still unconscious during conservative treatment period in ICU. On the nineteenth hospital day, our department participated in consultation. We believed that the patient had a pseudo-aneurysm of the subclavian artery complicated with vertebral arteriovenous malformation. We treated patient with selective arterial embolization on the second day.

At the beginning of the operation, we performed selective arteriography again , and found out that right subclavian artery covered stent failed to completely close the right vertebral artery, meanwhile, pseudo-aneurysm still developed(fig.4). Contralateral vertebral arteriography showed that contrast medium retrograde perfused the right vertebral artery, and arteriovenous fistula of right vertebral artery developed (fig.5). Super selected the pseudo-aneurysm by nerve micro catheter through the left vertebral artery to the right vertebral artery. Embolized pseudo-aneurysm with GDC through nerve micro catheter until the velocity of contrast medium was decreased. Applied Onyx to complete embolized the pseudo-aneurysm and fistula, preformed angiography again to confirm that no contrast medium was injected into the right vertebral artery (fig.6).

The patient's condition improved obviously after she returned to ICU, free movement, good sleep, no obvious respiratory symptoms and mental symptoms. Finally, she was discharged two weeks later.

The patient came hospital to reexamination, showed that the subclavian covered stent was mild stenosis, the right vertebral artery was occluded, and the right vertebral artery was not developed by the left vertebral artery angiography (fig.7).

\section{Discussion}

Pseudo-aneurysm is a kind of hematoma secondary to trauma and inflammation, it is distinguished from the aneurysm due to its lesion involving the whole layer of the blood vessel. Early detection and early diagnosis is the key to reduce the mortality of pseudo-aneurysm. ${ }^{14}$

DuBose conducted a review of the medical literature from 1990 to 2012 which reports documenting the use of endovascular stenting for the treatment of subclavian or axillary artery injuries. His statistics shows that $56.3 \%$ of penetrating injury cases received endovascular treatment, most of them are pseudo-aneurysm, furthermore, and the iatrogenic penetrating injury accounted for $21.8 \%$. In these cases, the success rate of stent implantation was $96.9 \%$, stent patency rate confirmed by imaging and clinical follow-up is $84.4 \%$ after 70 months. ${ }^{8}$ Endovascular embolization for the treatment of subclavian artery pseudo-aneurysm is an effective way, and it is safe and effective from reports of long-term follow-up. ${ }^{18}$ Iatrogenic subclavian artery injury is common in subclavian artery puncture, its complications are also abundant, including catheter malposition, vascular rupture, and pneumothorax. Mycotic aneurysms are also reported in a few cases, with an incidence of about $0.65-2 \% .^{5}$

This pseudo-aneurysm case is caused by acupuncture, which is very rare in reports, and it is very dangerous because the condition is difficult to detect. Due to the wide range of acupuncture treatment, there are many positions of false aneurysms caused by acupuncture in reports, likes body trunk and limbs. ${ }^{12}$ Its treatments are also depend on different conditions, locations, 
including detachable balloon, coil, liquid embolic agent embolization, stent implantation and other means. 9, 10, 17

Treatment of subclavian pseudo-aneurysm, in principle, recommend the use of endovascular therapy, according to most experience shows that endovascular treatment can significantly reduce the amount of intraoperative bleeding. ${ }^{15}$ In this case, the choice of endovascular stent treatment for this patient is more appropriate, however, the choice of stent diameter in the first treatment is still questionable. The diameter of the subclavian artery stent implantation is generally chose between $7-10 \mathrm{~mm}^{7}$. The standard of successful stent implantation should be the correct choice of stent or stop bleeding, the recovery of distal blood flow, no reperfusion injury and long-term patency of the stent in clinical. ${ }^{4}$ Taking into account the subclavian artery of patients was in bleeding spastic period, with severe systemic bleeding, despite the measurement of vascular diameter about $6 \mathrm{~mm}$, it is recommended to choose a slightly larger diameter covered stent, that is the deficiency of treatment for the first time. Attention should be paid to the position relation between the vertebral artery and the injury of the blood vessels in the stent placement, if the vertebral artery is close to the false aneurysm, it is easy to cause the occlusion of the vertebral artery. ${ }^{1}$ But the patient had arteriovenous malformation of the vertebral artery simultaneously. By angiography, we can see that the pseudo-aneurysm is associated with the vascular malformation, and relevant vein developed. (fig.5)After patient awake, we communicate with her also confirmed that patient having consciously right neck discomfort, so she used acupuncture to relieve symptoms for a long time. The patient is complicated by the complexity of her condition and should be given priority in the embolization of pseudo-aneurysm and arteriovenous malformations. Vascular injury can be blocked by GDC or balloon occlusion. Because the patient's right subclavian artery route was covered with a covered stent, we tried go through it unsuccessfully, so we diverted into the right vertebral artery malformation by retrograde pass through left subclavian vascular. By reaching the lesion in retrograde route, we use spring coils to slow the flow of blood, and use Onyx to compact embolize the lesion, so as to achieve the purpose of closing the lesion. Endovascular treatment of subclavian artery pseudo-aneurysm also include the brachial artery approach, the advantages lie in that path is simple and convenient, but due to the limitations of vessel sheath diameter, whether apply this way should be based on intraoperative condition. ${ }^{1}$

Compared with endovascular treatment, surgical treatment of subclavian lesions has been the primary choice of the doctor all the time. In recent years, with the discussions about the difficulty of operation, intraoperative trauma, significant complications and etc., more and more people have recognized the advantages of endovascular interventional treatment in various traumatic lesions. 1,3,4 However, due to the limitations of medical technology, there are many differences in the endovascular treatment therapeutic effect among different doctors, so many people still have doubts about the choice of interventional therapy. ${ }^{6}$ According to a meta-analysis, despite the theoretical advantages of interventional treatment of vascular trauma is very large, it's the clinical evidence is poor, the durability and long-term effect of the stent to be verified. ${ }^{16}$ As a doctor there is still a long way to explore and endeavor in the future.

\section{Conclusion}

Due to the anatomic relationship of bilateral vertebral artery, we suggest that the contralateral vertebral artery can be used to reach the lesion when one side of the vertebral artery disease is extremely difficult to pass.

\section{References}

1. Aalami OO, Lisagor P, Victorino GP, et al. Repair of Iatrogenic Subclavian Artery Pseudoaneurysm with Covered Stent Placement after Vertebral Artery Transposition. Journal of Vascular and Interventional Radiology. 2005;16(12):1759-1760.

2. Bergqvist D. Vascular injuries caused by acupuncture. 
European journal of vascular and endovascular surgery : the official journal of the European Society for Vascular Surgery. 2008;36(2):160-163.

3. Branco BC, Boutrous ML, DuBose JJ, et al. Outcome comparison between open and endovascular management of axillosubclavian arterial injuries. Journal of Vascular Surgery. 2016;63(3):702-709.

4. Carrafiello GL, Domenico Mangini, Monica Fontana, Federico Chiara, Recaldini Filippo, Piacentino Carlo, Pellegrino Piffaretti, Gabriele, Fugazzola C. Percutaneous Treatment of Traumatic Upper-extremity Arterial Injuries: A Single-center Experience. Journal of Vascular and Interventional Radiology. 2011;22(1):34-39.

5. Chen Y-F, Su C-S, Liu T-J, et al. Endovascular treatment of a nontraumatic left subclavian artery pseudoaneurysm. Journal of the Chinese Medical Association. 2012;75(9):474-478.

6. Chong VE, Lee WS, Miraflor E, et al. Applying peripheral vascular injury guidelines to penetrating trauma. Journal of Surgical Research. 2014;190(1):300-304.

7. du Toit DF, Lambrechts AV, Stark H, et al. Long-term results of stent graft treatment of subclavian artery injuries: Management of choice for stable patients? Journal of Vascular Surgery. 2008;47(4):739-743.

8. DuBose JJ, Rajani R, Gilani R, et al. Endovascular management of axillo-subclavian arterial injury: A review of published experience. Injury. 2012;43(11):1785-1792.

9. Ember L. Ewings M, Catherine M. Wittgen, MD, and Christian E. Paletta, MD. Prolonged Success With a Covered Endovascular Stent After Emergent Use in Radiation-Induced Subclavian Artery Blowout: A Case Report. Vascular and Endovascular Surgery. 2008;42(2).

10. Hizette P, Horn D, Lemaitre J, et al. Endovascular repair of a life-threatening radiation-induced ruptured false aneurysm of the intrathoracic left subclavian artery: case report. Vascular health and risk management. 2015;11:219-222.

11. Mammen S, Koshy G, Khurana A, et al. Endovascular repair of iatrogenic subclavian artery pseudo-aneurysm. Saudi Journal of Kidney Diseases and Transplantation. 2015;26(5):1023-1025.

12. Matsuyama H, Nagao K, Yamakawa G-I, et al. RETROPERITONEAL HEMATOMA DUE TO RUPTURE OF A PSEUDOANEURYSM CAUSED BY ACUPUNCTURE THERAPY. The Journal of Urology. 1998;159(6):2087-2088.

13. Michaud K, Grabherr S, Lesta Mdel M, et al. Ruptured pseudo-aneurysm of a femoral artery in a drug abuser revealed by post-mortem CT angiography. International journal of legal medicine. 2013;127(4):819-823.

14. Minghao Lu B, Clifford Weiss, MD, Elliot K. Fishman, MD, Pamela T. Johnson, MD, and Franco Verde, MD. Review of Visceral Aneurysms and Pseudoaneurysms. J Comput Assist Tomogr. 2015;39(1).

15. Scott AR, Gilani R, Tapia NM, et al. Endovascular management of traumatic peripheral arterial injuries. Journal of Surgical Research. 2015;199(2):557-563.

16. Sinha $\mathrm{S}$, Patterson BO, Ma J, et al. Systematic review and meta-analysis of open surgical and endovascular management of thoracic outlet vascular injuries. Journal of Vascular Surgery. 2013;57(2):547-567.e548.

17. Wozniak W, Mlosek RK, Gorski G, et al. Thrombin obliteration of subclavian artery aneurysm with intravascular balloon protection. Kardiochirurgia $\mathrm{i}$ torakochirurgia polska $=$ Polish journal of cardio-thoracic surgery. 2014;11(2):202-204.

18. Zehm S, Chemelli A, Jaschke W, et al. Long-term outcome after surgical and endovascular management of true and false subclavian artery aneurysms. Vascular. 2014;22(3):161-166. 
Interventional Treatment of Iatrogenic False Aneurysm of Subclavian Artery Combine with Arteriovenous Malformation

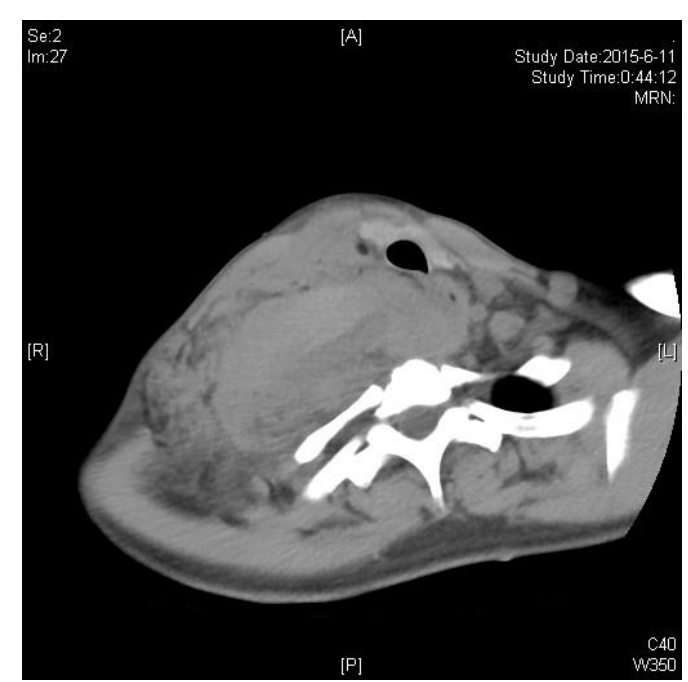

fig 2

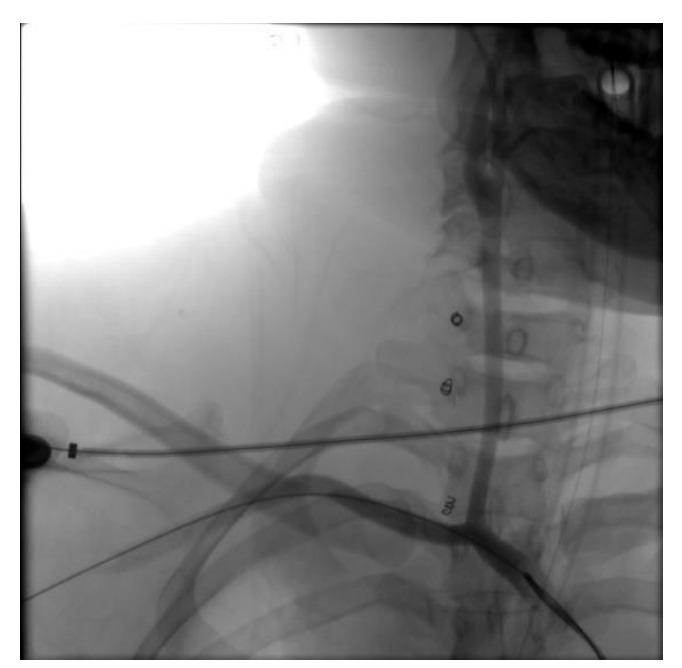

fig 3

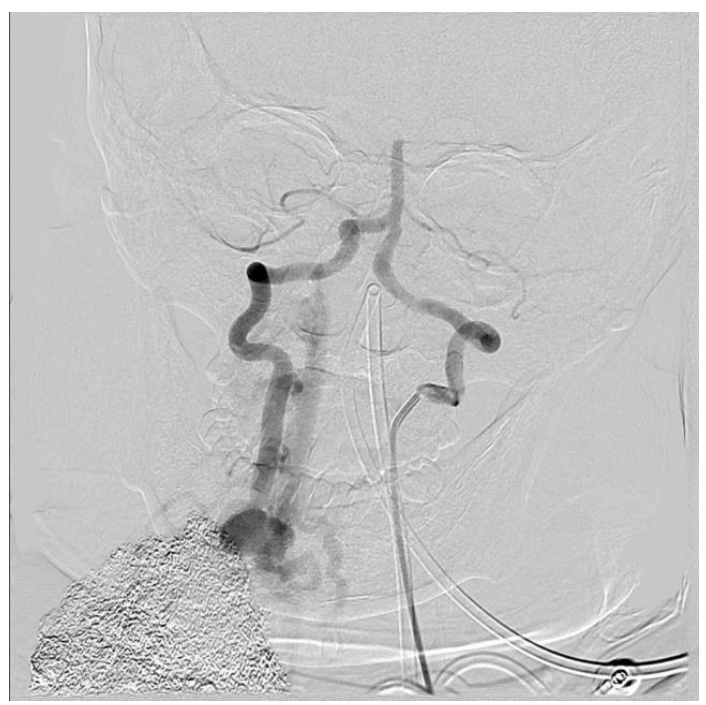

fig 6

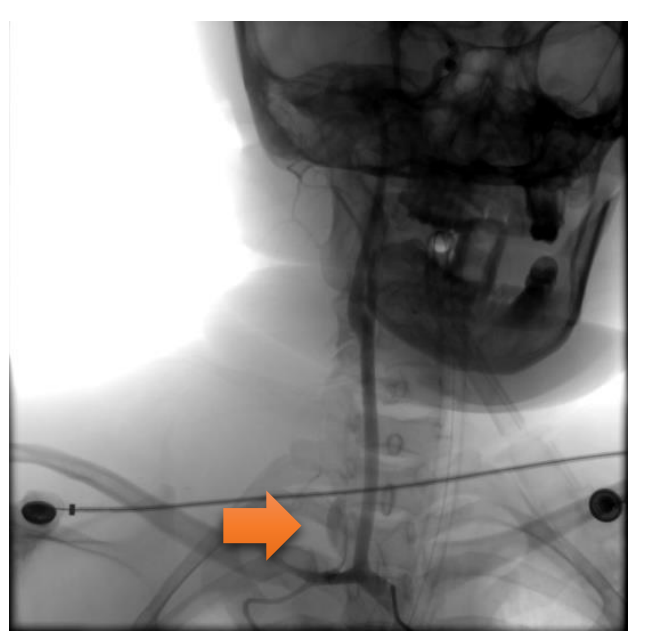

fig 1

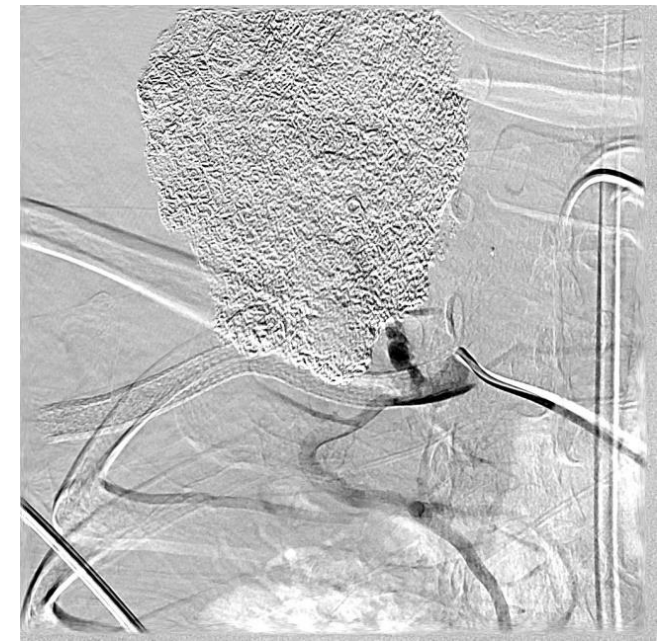

fig 4

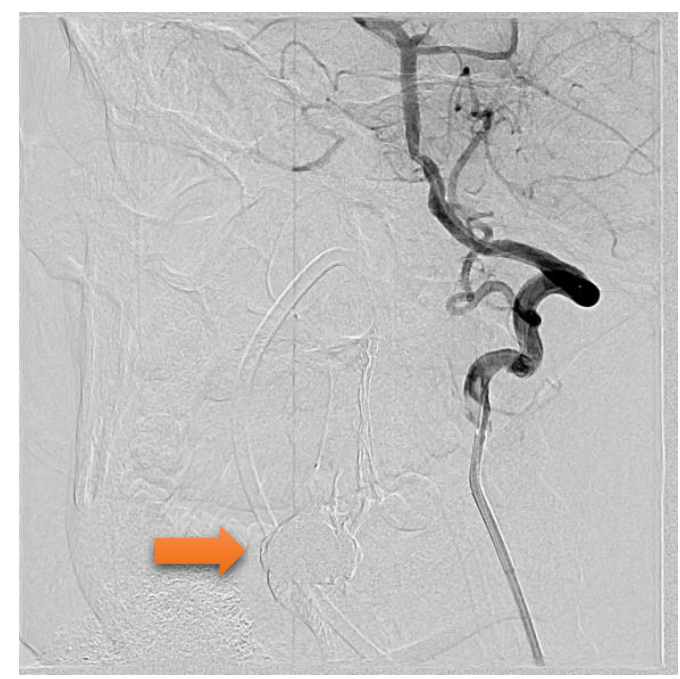

fig 5 


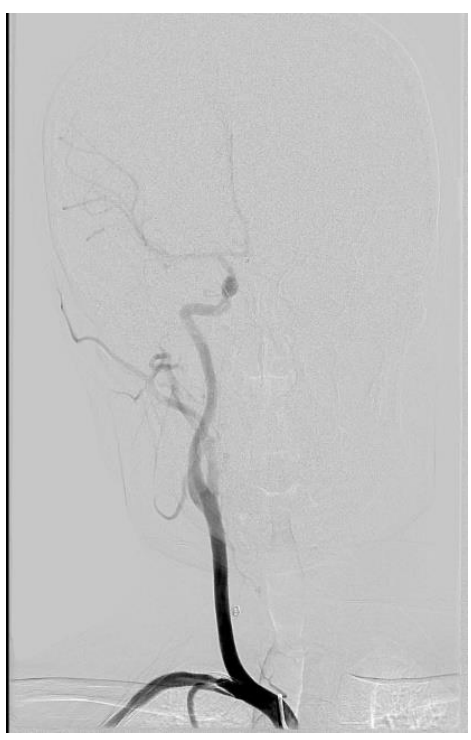

fig 7 